\title{
Subcorneal pustular dermatosis in the pediatric age*
}

\author{
Márcio Martins Lobo Jardim ${ }^{1}$ \\ Thaís Amaral Carneiro Cunha ${ }^{1}$
}

\author{
Ticiana Andrade Castelo Branco Diniz ${ }^{1}$ \\ Neusa Yurico Sakai Valente ${ }^{1}$
}

DOI: http:/ /dx.doi.org/10.1590/abd1806-4841.20186576

\begin{abstract}
Subcorneal pustular dermatosis is a rare pustular eruption which occurs mainly in middle-aged women and rarely during childhood. We report a case of a 15-year-old female with a 4-year history of pustular lesions on the proximal region of the upper limbs with subsequent impairment of the trunk. Physical examination revealed small pustules distributed on the trunk and proximal region of the limbs. Histopathology showed a subcorneal pustule and direct immunofluorescence for IgA, IgM, IgG and fibrinogen was negative, confirming the diagnosis of subcorneal pustular dermatosis. The patient was treated with dapsone with good clinical response after one month. Subcorneal pustular dermatosis is a rare condition and there are only isolated cases reported in the literature in pediatric patients. Thus, we discuss the main clinical aspects and treatment response of this condition during childhood.
\end{abstract}

Keywords: Dapsone; ; Pediatrics; Skin diseases, vesiculobullous

\section{INTRODUCTION}

Subcorneal pustular dermatosis (SPD), also known as Sneddon-Wilkinson's disease, is a rare, chronic, relapsing pustular eruption. ${ }^{1}$ It is clinically relevant given its association with malignant neoplasms such as multiple myeloma, chronic lymphocytic leukemia and thymoma. ${ }^{2}$

This condition is more common in women over 40 years of age and few cases have been reported in children. ${ }^{3}$ We report a case of a teenager diagnosed with SPD.

\section{CASE REPORT}

A 15-year-old female patient presented with a 4-year history of pustular lesions on the proximal region of the upper limbs with a subsequent impairment of the chest and abdomen. During this period, the lesions showed temporary episodes of improvement that evolved with residual hyperchromic macules.

Physical examination revealed pustular lesions with a slightly erythematous base, isolated or grouped in a serpiginous pattern, on the trunk and proximal region of the upper and lower limbs. Hyperchromic macules were observed mingled with pus- tular lesions. There were no lesions on the face, palms, soles, and mucous membranes (Figures 1 and 2). A complete blood count, serum protein electrophoresis and liver, kidney and thyroid functions showed normal results.

Histopathology revealed a subcorneal spongiform pustule (Figure 3). Direct immunofluorescence of the perilesional skin for $\operatorname{IgA}$, IgM, IgG and fibrinogen was negative, thus excluding $\operatorname{Ig} \mathrm{A}$ pemphigus.

Considering the clinical and histopathological findings, we confirmed the diagnosis of SPD. The patient was treated with dapsone $(100 \mathrm{mg} /$ day) with a significant improvement after one month, showing only hyperchromic macules and residual crusts (Figure 4). After treatment was instituted we observed a slight increase of total bilirubin $(\mathrm{TB}=1.7, \mathrm{DB}=1.2)$ and a slight decrease of hemoglobin $(\mathrm{Hb}$ $=10.7$ ). There were no alterations on the remaining markers of hemolysis and on G6PD dosage. We chose to maintain the treatment and throughout the patient's follow-up all laboratory results remained stable. After complete clinical improvement, dapsone was tapered to $50 \mathrm{mg} /$ day and the patient was followed-up every three months. Nine months after treatment was started no flares had been noticed.

Received on 07.10.2016.

Approved by the Advisory Board and accepted for publication on 31.01.2017.

* Work preformed at the Hospital do Servidor Público Estadual de São Paulo.

Financial support: none.

Conflict of interest: none.

1 Department of Dermatology. Hospital do Servidor Público Estadual de São Paulo - São Paulo (SP), Brazil.

MAILING ADDRESS:

Márcio Martins Lobo Jardim

E-mail: martinslobojardim@gmail.com 


\section{DISCUSSION}

SPD is a rare condition with few published case reports.. It was first described by Sneddon and Wilkinson in 1956 when the authors reported six cases of the disease, five of which in women between 40 and 68 years old. ${ }^{1}$ Since then, case reports have confirmed that this disease occurs more frequently in women of this age group, and few studies have reported the disease in children or teenagers.

The first case of pediatric SPD was reported in 1958 by Sakany et al. in a 10-year-old patient. ${ }^{3}$ Later in 1974, a case report of two children with a classic clinical presentation was published, however, only one child responded well to dapsone. ${ }^{4}$ Since then, the case

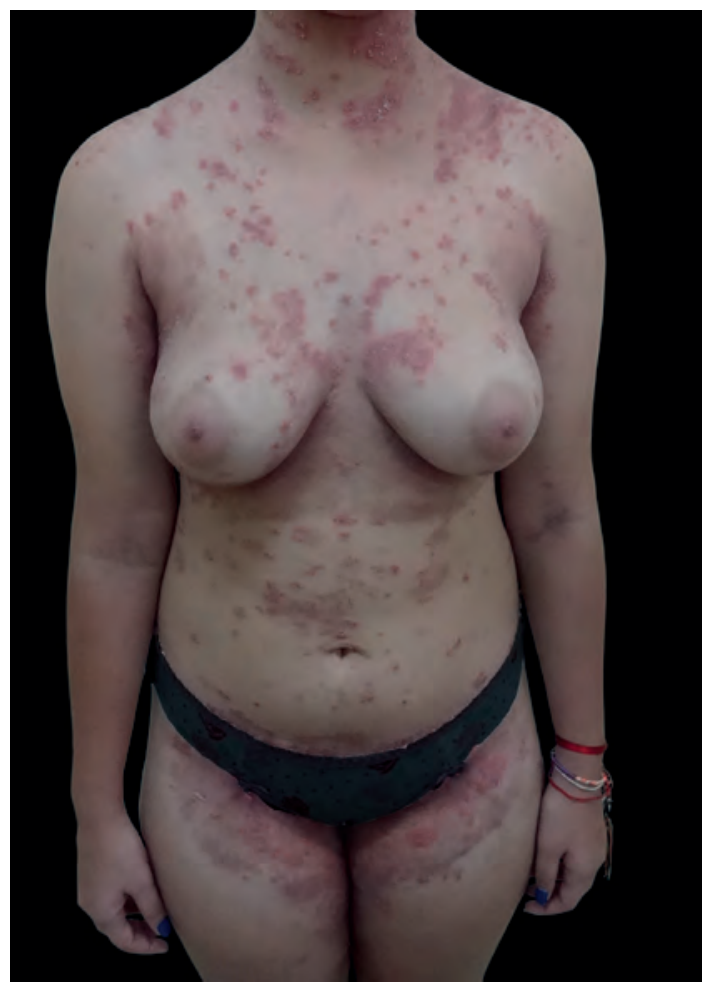

FIGURE 1: Before treatment: pustular eruption distributed throughout the trunk and flexor aspects of the limbs mingled with hyperchromic macules

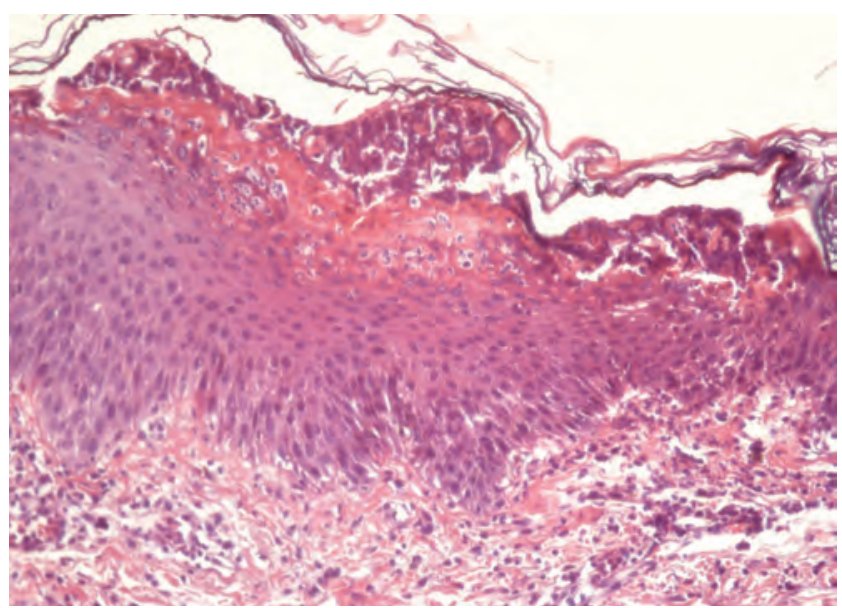

FiguRE 3: Subcorneal spongiform pustule (Hematoxylin \& eosin, X200) reports of pediatric SPD have indicated that the clinical condition is similar both in children and adults. In 2013, Scalvenzi et al. reported a case of a 7-year-old patient with an erythematous-base pustular

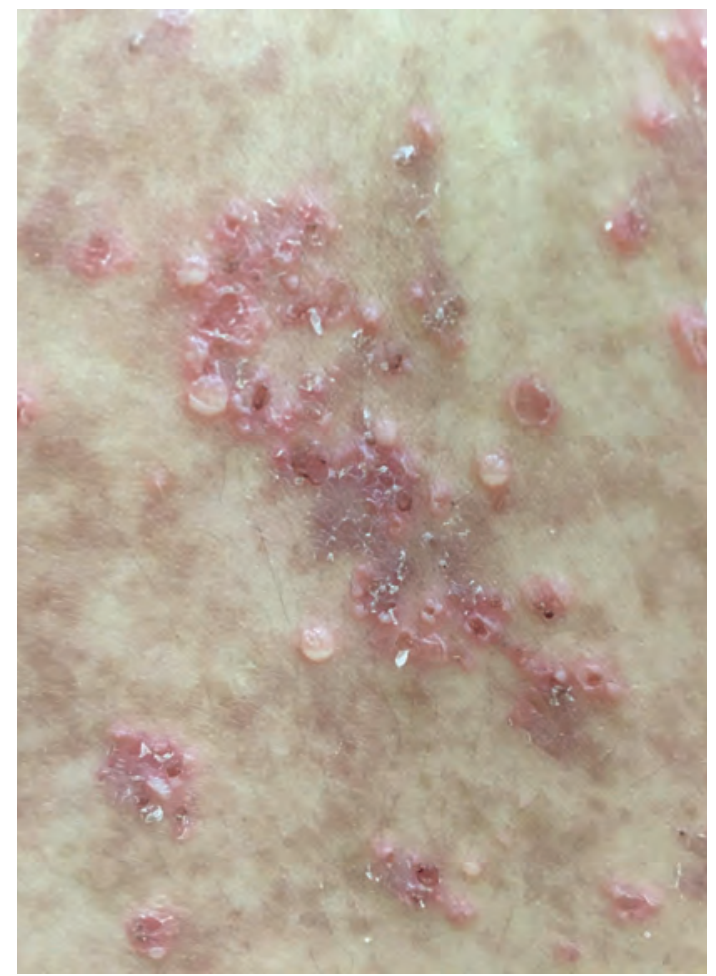

FIGURE 2: The "half-and-half" aspect of pustules with a serpiginous aspect, erythematous base and crusts

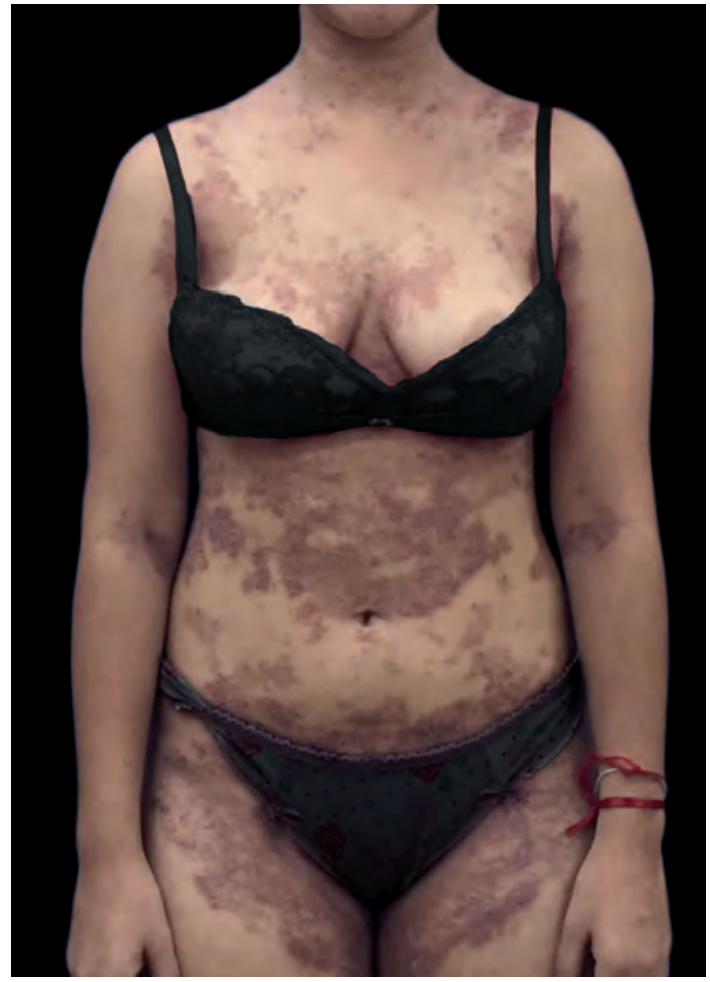

FIGURE 4: After treatment (30 days): hyperchromic macules in the regions of previous pustular lesions 
eruption for three weeks and described it as "half-and-half", indicating that the lower half of the lesion presented a sterile purulent content and the upper half a serous one, which is characteristic of the disease. ${ }^{1}$ There are less than 20 cases of pediatric SPD described in the literature. ${ }^{5-10}$

Histopathology of SPD does not show pathognomonic findings; however, it does reveal subcorneal pustules and dermal infiltrate containing mainly neutrophils with few eosinophils, little or no spongiosis, and rarely acantholysis. Characteristically, direct immunofluorescence studies are negative. The differential diagnosis of SPD includes pustular psoriasis, IgA pemphigus, pemphigus foliaceus, dermatitis herpetiformis, impetigo, and acute generalized exanthematous pustulosis (AGEP). ${ }^{2}$

Despite the well documented association of SPD with multiple myeloma, IgA monoclonal gammopathy, chronic lymphocytic leukemia, thymoma, rheumatoid arthritis and thyroid disorders, our patient did not show any clinical symptom or laboratory result of systemic diseases during follow-up.

\section{REFERENCES}

1. Sneddon IB, Wilkinson DS. Subcorneal pustular dermatosis. Br J Dermatol. 1956:68:385-94.

2. Cheng S, Edmonds E, Ben-Gashir M, Yu RC. Subcorneal pustular dermatosis: 50 years on. Clin Exp Dermatol. 2008;33:229-33

3. Sarkany I. Subcorneal pustular dermatosis. Br J Dermatol. 1958;70:307.

4. Johnson SA, Cripps DJ. Subcorneal pustular dermatosis in children. Arch Dermatol. 1974:109:73-7.

5. Scalvenzi M, Palmisano F, Annunziata MC, Mezza E, Cozzolino I, Costa C. Subcorneal Pustular Dermatosis in Childhood: A case report and review of the literature. Cases Reports InDermatological medicine. 2013; 424797.

6. Tosi, V, Buján MM, Merediz J, Andrea B, Pierini A. Pustulosis subcórnea de Sneddon-Wilkinson. Arch Argent Dermatol. 2009;59:159-63.

7. Reed J, Wilkinson J. Subcorneal pustular dermatosis. Clin Dermatol. 2000;18:301-13
The first choice of treatment in children is dapsone 100$150 \mathrm{mg} /$ day, similar to what is indicated for adults. However, cases of hematologic toxicity to the drug have been reported in children, mainly hemolytic anemia and methemoglobinemia. In 2003, Koçak et al. observed a decrease in hemoglobin in a child with SPD treated with dapsone for three weeks, which normalized after the drug was withdrawn. In 2009, Tosi et al. published two cases of pediatric SPD in which one child manifested methemoglobinemia when treated with dapsone, which was also reversed once the drug was discontinued. ${ }^{6,9}$

Even though our patient showed a slight decrease in hemoglobin and a slight increase in bilirubin, her laboratory results and clinical evaluations remained stable throughout monthly follow-ups. Thus, there was no need to reduce the dosage of the drug before complete clinical improvement. Although dapsone remains the first choice of treatment, a rigorous clinical and laboratorial surveillance is needed in order to detect the first manifestations of its toxicity. Other therapeutic options for children are topical or systemic corticosteroids and acitretin. ${ }^{6,9}$

8. Koçak M, Birol A, Erkek E, Bozdoğan 0, Atasoy P. Juvenile subcorneal pustular dermatosis: a case report. Pediatr Dermatol. 2003:20:57-9.

9. Yayli S, Bahadir S, Alpay K, Cimşit G, Reis A. A case of Juvenile subcorneal pustular dermatosis successfully treated with acitretin. Int J Dermatol. 2006;45:1131-3.

10. Watts PJ, Khachemoune A. Subcorneal Pustular Dermatosis: A Review of 30 Years of Progress. Am J Clin Dermatol. 2016;17:653-671.

How to cite this article: Lobo-Jardim MM, Diniz TACB, Cunha TAC, Valente NYS. Subcorneal pustular dermatosis in the pediatric age. An Bras Dermatol. 2018;93(1):116-8. 